https://helda.helsinki.fi

\title{
Gravitational waves from holographic neutron star mergers
}

\section{Ecker, Christian}

2020-05-05

Ecker , C , Järvinen , M , Nijs , G \& van der Schee , W 2020 , ' Gravitational waves from holographic neutron star mergers ' , Physical Review D , vol. 101 , no. 10 , 103006 . https://doi.org/10.1103/PhysRev

http://hdl.handle.net/10138/315214

https://doi.org/10.1103/PhysRevD.101.103006

unspecified

publishedVersion

Downloaded from Helda, University of Helsinki institutional repository.

This is an electronic reprint of the original article.

This reprint may differ from the original in pagination and typographic detail.

Please cite the original version. 


\title{
Gravitational waves from holographic neutron star mergers
}

\author{
Christian Ecker $\odot,{ }^{1,2, *}$ Matti Järvinen, ${ }^{1,3, \dagger}$ Govert Nijs $\odot,{ }^{1, \$}$ and Wilke van der Schee ${ }^{1, \S}$ \\ ${ }^{1}$ Institute for Theoretical Physics and Center for Extreme Matter and Emergent Phenomena, Utrecht \\ University, Leuvenlaan 4, 3584 CE Utrecht, The Netherlands \\ ${ }^{2}$ Instituut-Lorentz, D-ITP, Universiteit Leiden, P.O. Box 9506, 2300 RA Leiden, The Netherlands \\ ${ }^{3}$ Department of Physics and Helsinki Institute of Physics, University of Helsinki, \\ P.O. Box 64, FI-00014 Helsinki, Finland
}

(Received 19 August 2019; accepted 15 April 2020; published 5 May 2020)

\begin{abstract}
We present simulations of binary neutron star mergers with equations of state (EoSs) that have input from holography, and analyze the spectral properties of the resulting waveforms. These EoSs consist of a standard nuclear matter EoS at low densities, transitioning to a state-of-the-art holographic EoS with firstorder deconfinement phase transition in the otherwise intractable high-density regime. Depending on the transition density, the characteristic frequencies in the spectrum produced from the hybrid EoSs are shifted to significantly lower values. Equal-mass binaries with a total mass of $2.8 M_{\odot}$ reach densities in the quark matter phase in the core of the transient hypermassive neutron star, which then induces an immediate gravitational collapse.
\end{abstract}

DOI: 10.1103/PhysRevD.101.103006

\section{INTRODUCTION}

Gravitational waves (GWs) provide us a direct view on the most violent events in our Universe [1,2], among which there will be tens of neutron star mergers in the near future. While general relativity (GR) is by now a well-understood theory, the merger of two neutron stars is clouded with considerably more uncertainty (see Ref. [3] for an excellent review). One profound reason is that the fundamental theory of quarks and gluons (QCD) is strongly coupled, and the dense state of neutron stars is inaccessible to the lattice. In this paper we hence use a strongly coupled (holographic) model and study its implications on the power spectral density (PSD) of GWs after a neutron star merger.

Our current knowledge about the equation of state $(\mathrm{EoS})$ in neutron stars comes from the low-density region (with energy density $\lesssim 0.2 \mathrm{GeV} / \mathrm{fm}^{3}$ ) where it is constrained by nuclear physics [4], and at very high densities $\left(\gtrsim 10 \mathrm{GeV} / \mathrm{fm}^{3}\right)$ where QCD is perturbative [5]. At intermediate densities not much is certain, but the EoS can be constrained by limiting the speed of sound as well as observations of mass-radius relationships and recently by the bound on the tidal deformability coming from the GW170817 merger measured by LIGO/Virgo [2]. Applying these constraints, it is likely that a phase transition is present [6], which may indicate the

\footnotetext{
*c.ecker@uu.nl

†.o.jarvinen@uu.nl

g.h.nijs@uu.nl

\$.vanderschee@uu.nl
}

presence of quark matter at high densities (see also Refs. [7-10]).

Since QCD at intermediate densities is strongly coupled, holography can be used to obtain qualitative insights. We use a holographic model to derive EoSs that satisfy the aforementioned astrophysical constraints and are then used as input in simulations of equal-mass neutron star mergers performed with the Einstein Toolkit [11]. Especially interesting are mergers of intermediate-mass stars $\left(M \approx 1.3-1.4 M_{\odot}\right)$ because they can lead to the formation of a metastable hypermassive neutron star (HMNS), whose gravitational-wave signal encodes characteristic information on the EoS [12-15].

Finally, we show how the PSD depends on both the parameters of our equation of state and the neutron star mass, and comment on how this compares with other EoSs. Future measurements by Advanced LIGO and the Einstein Telescope will be able to experimentally distinguish these different EoSs (see also Refs. [12,13] for earlier work with traditional nuclear matter EoSs).

\section{HOLOGRAPHIC MODEL}

The holographic model (V-QCD), which we use to describe strongly interacting dense nuclear and deconfined quark matter [16], is obtained through a fusion of two building blocks: improved holographic QCD [17], a bottom-up model for the gluon sector inspired by five-dimensional noncritical string theory, and a framework based on tachyonic D-brane actions for the flavor sector $[18,19]$. Details of the model are chosen to reproduce a number of features of QCD. This includes linear confinement, 
asymptotic freedom, chiral symmetry breaking, a qualitatively reasonable hadron spectrum, and a finite-temperature phase diagram with structure as expected from QCD [1623]. The thermodynamics is tuned to match lattice data at temperatures right above the confinement/chiral transition $[24,25]$.

This model has a nuclear phase [26] and a deconfined quark matter phase $[25,27,28]$ at low and high densities, respectively. For the nuclear phase (essential for neutron stars), a simple method is used $[26,29,30]$ that approximates nuclear matter as a homogeneous field. The EoS for dense nuclear matter is stiff in this approach (i.e., the speed of sound is relatively high) and depends, among other things, on the coupling $b$ between the baryons and the chiral condensate (see Ref. [26] for a complete exposition).

\section{HYBRID EQUATIONS OF STATE}

The weakly coupled, low-density regime of cold nuclear matter is better described by using traditional effective methods. Therefore, we construct "hybrid" equations of state composed of i) the SLy EoS [31,32] for low-density nuclear matter, and ii) the V-QCD EoS for dense nuclear and quark matter described in Sec. II. We choose the matching density $\rho_{m}$ between SLy and V-QCD to lie at 1.5-2 times the nuclear saturation density $\rho_{s}=2.04 \times 10^{14} \mathrm{~g} / \mathrm{cm}^{3}$, and then fix $b$ as well as the normalization of the baryon action $c_{b}$ by requiring that the pressure and its derivative with respect to the chemical potential are continuous at the matching point.

Basic properties of the obtained hybrid EoSs and transitions for several choices of $\rho_{m}$ are listed in Table I. The speed of sound $c_{s}$ of the holographic EoS depends only weakly on $b$ and is higher than that of SLy. Note also that $\rho_{m}$ depends monotonically on $b$, leading to a more holographic EoS as $b$ decreases. One can then see in Fig. 1 that the higher holographic $c_{s}$ leads to a higher pressure, and hence increasingly stiff EoSs as $b$ is decreased. Figure 2 then shows the corresponding mass-radius relation obtained from solving the Tolman-Oppenheimer-Volkoff equations.

Our hybrid EoSs do not support quark matter cores, since the branch with central density larger than $\rho_{\mathrm{QM}}$ (thin lines in Fig. 2 left of the kinks at the maximum masses) are unstable [34]. Reference [6] argued that NSs without quark matter

TABLE I. Properties of irrotational neutron stars with hybrid SLy + V-QCD and the SLy EoSs. Here $\rho_{\mathrm{NM}}\left(\rho_{\mathrm{QM}}\right)$ is the nuclear (quark) matter density at the transition.

\begin{tabular}{lcccccc}
\hline \hline$\frac{\rho_{m}}{\rho_{s}}$ & $b$ & $\frac{\rho_{\mathrm{NM}}}{\rho_{s}}$ & $\frac{\rho_{\mathrm{QM}}}{\rho_{s}}$ & $\frac{M_{\max }}{M_{\odot}}$ & $R\left(1.4 M_{\odot}\right)[\mathrm{km}]$ & $\Lambda\left(1.4 M_{\odot}\right)$ \\
\hline 1.44 & 10.45 & 4.29 & 7.93 & 2.25 & 13.0 & 680 \\
1.61 & 10.50 & 4.83 & 8.57 & 2.17 & 12.6 & 550 \\
1.77 & 10.55 & 5.37 & 9.24 & 2.10 & 12.3 & 470 \\
1.94 & 10.60 & 5.86 & 9.79 & 2.04 & 12.1 & 410 \\
2.10 & 10.65 & 6.23 & 10.21 & 2.00 & 12.0 & 370 \\
SLy & $\cdots$ & $\cdots$ & $\cdots$ & 2.05 & 11.7 & 300 \\
\hline \hline
\end{tabular}

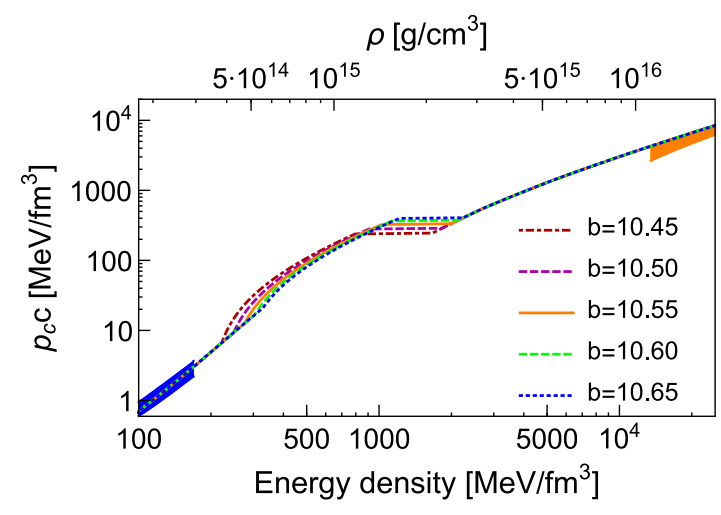

FIG. 1. The constructed family of the hybrid EoSs. Blue and orange regions show the error bands for QCD from effective Lagrangians and perturbative QCD, respectively. The $b$ dependence on the $\rho$ axis (which is a nontrivial function of the energy density) is not visible in the plot.

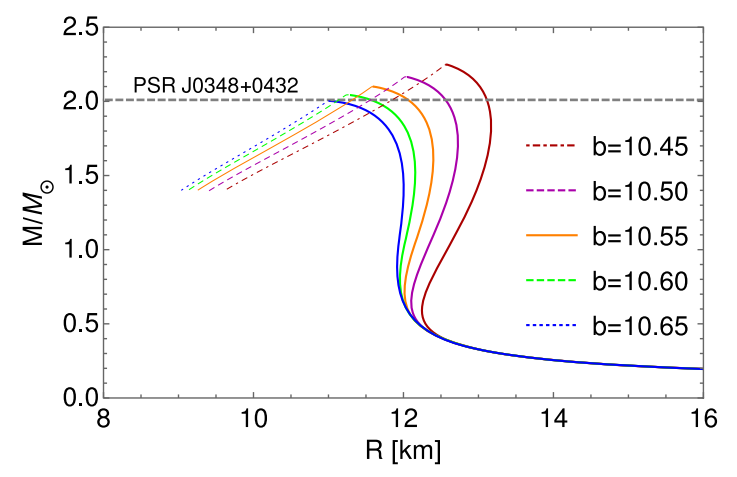

FIG. 2. Mass-radius relation for different values of $b$ together with the observational lower mass bound of Ref. [33].

cores and masses consistent with the current observational bounds are only possible for speeds of sound well above the conformal value, $c_{s}^{2}>1 / 3$. Our setup provides an explicit realization of such a scenario, as $c_{s}^{2}$ is significantly larger than $1 / 3$ over a wide range of chemical potentials in the holographic baryon phase [26]. Moreover, our NS radii are approximately $12-13 \mathrm{~km}$, which is consistent with recent measurements of $\mathrm{x}$-ray bursts from binary systems $[35,36]$.

Our choices in Table I scan over the physically allowed EoSs. Indeed, the first tabulated hybrid $\operatorname{EoS}(b=10.45)$ is already too stiff: the value of the tidal deformability $\Lambda \simeq$ 680 for a neutron star with mass $\simeq 1.4 M_{\odot}$ is well above the bound set by LIGO [37] that $\Lambda \lesssim 580$ (at the $90 \%$ confidence level). Similarly, the maximum mass $M_{\max }$ for the last hybrid $\operatorname{EoS}(b=10.65)$ is below the best estimate of the observed mass of the pulsar PSR J0348+0432 $\left(M=2.01 \pm 0.04 M_{\odot}\right)[33]$ and the mass of PSR J0740+ $6620\left(M=2.14_{-0.09}^{+0.10} M_{\odot}\right)[38]$. We choose to use the EoSs with $b=10.5$ and $b=10.6$, which are consistent with the astrophysical bounds by a clear margin. 
We account for shock-heating effects [39] during the merger by adding to the cold holographic EoS $p_{\mathrm{c}}(\rho)$ a thermal component $p_{\text {th }}=\Gamma_{\text {th }} \rho\left(\epsilon-\epsilon_{\mathrm{c}}\right)$ with $\Gamma_{\text {th }}=1.75$, where $\rho \equiv m_{b} n_{b}$ is the rest-mass density, $m_{b}$ is the baryon mass, and $n_{b}$ is the number density. This gives a total pressure $p=p_{c}+p_{\text {th }}$ and specific internal energy $\epsilon=\epsilon_{c}+\epsilon_{\text {th }}$.

\section{NUMERICAL SETUP}

We use the LORENE pseudospectral code [40] to generate initial data for two irrotational stars of equal mass $M$ on quasicircular orbits with a diameter of $45 \mathrm{~km}$, where $M$ is the gravitational mass of the isolated star. These initial data give approximately three $\left(M=1.5 M_{\odot}\right)$ to six $\left(M=1.3 M_{\odot}\right)$ orbits before merger. The initial data are evolved by solving the $3+1$-dimensional Einstein equations coupled to ideal general-relativistic hydrodynamics (GRHD) [41] using the Einstein Toolkit [42-44].

The GRHD equations are solved in conservative form [45] using the high-order, high-resolution shock-capturing code WHISKYTHC [46-50]. All simulations have a volume of size $\approx 3025^{3} \mathrm{~km}^{3}$ and assume radiative and static boundary conditions for the metric and the hydrodynamic variables, respectively, as well as reflection symmetry across the $z=0$ plane. Our meshes have six refinement levels with a finest resolution of $368 \mathrm{~m}$ covering the individual stars and the merger remnant. We follow Ref. [12] and compute the PSD of the dominant $\ell=m=$ 2 modes $h_{+, x}^{22}$ via

$$
\tilde{h}(f) \equiv \sqrt{\frac{\left|\int h_{+}^{22}(t) e^{-i 2 \pi f t} d t\right|^{2}+\left|\int h_{\times}^{22}(t) e^{-i 2 \pi f t} d t\right|^{2}}{2}}
$$

where we take the time interval between -7 and $24 \mathrm{~ms}$ and define $t=0$ by the maximum of the GW amplitude. Motivated by the estimated luminosity distance of GW170817 [2], we extrapolate the GW signal in all of our simulations to an assumed detector distance of $40 \mathrm{Mpc}$.

\section{RESULTS}

In Fig. 3 we show snapshots of the rest-mass density $\rho$ in the orbital plane and the corresponding waveform for a binary merger with individual mass $M=1.3 M_{\odot}$ for our hybrid EoS with $b=10.5$ [51]. The first plot $(t=-1.6 \mathrm{~ms})$ shows a late stage of the inspiral, where the mutual tidal deformations of the stars become significant and full numerical GR simulations become important. The second plot $(t=4.3 \mathrm{~ms}$ ) shows an early post-merger stage, where the rotating and oscillating merger remnant is bar deformed and still nonaxially symmetric. This phase lasts for about $10 \mathrm{~ms}$ and the corresponding GW signal contains spectral features that are sensitive to the EoS, which we will analyze below. The third plot $(t=30 \mathrm{~ms})$ shows the late post-merger stage, where the merger remnant has settled down to an almost axially symmetric rotating star with a lifetime $>40 \mathrm{~ms}$. The $M=1.4 M_{\odot}$ case (Fig. 4) results in a relatively short-lived, highly deformed HMNS, which collapses $\approx 7.8 \mathrm{~ms}$ after the merger into a black hole with a torus of remaining infalling matter. The increased mass density after the phase transition results in prompt collapse to a black hole, providing an example for the phase-transition-triggered collapse scenario introduced in Ref. [52]. This sudden collapse and the corresponding imprint on the waveform
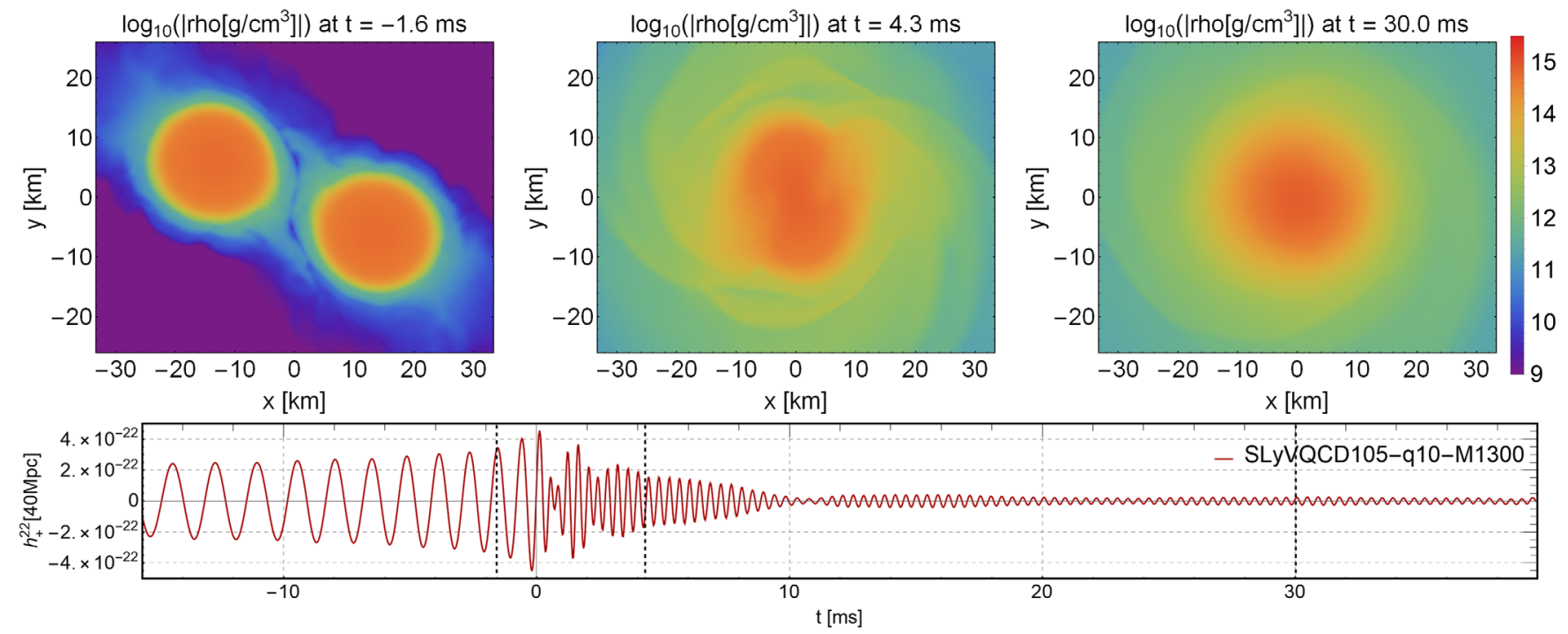

FIG. 3. Top: Snapshots of the rest-mass density in the orbital plane for the late inspiral phase (left), the early post-merger phase (center), and the late post-merger phase (right) for the $M=1.3 M_{\odot}$ equal-mass binary and holographic EoS with $b=10.5$. Bottom: GW strain signal at an assumed detector distance of $40 \mathrm{Mpc}$, i.e., the estimated luminosity distance of GW170817 [2]. The label SLyVQCD105-q10-M1300 refers, respectively, to the equation of state, the mass ratio (1.0), and the average mass of the stars (1.3 solar masses). 

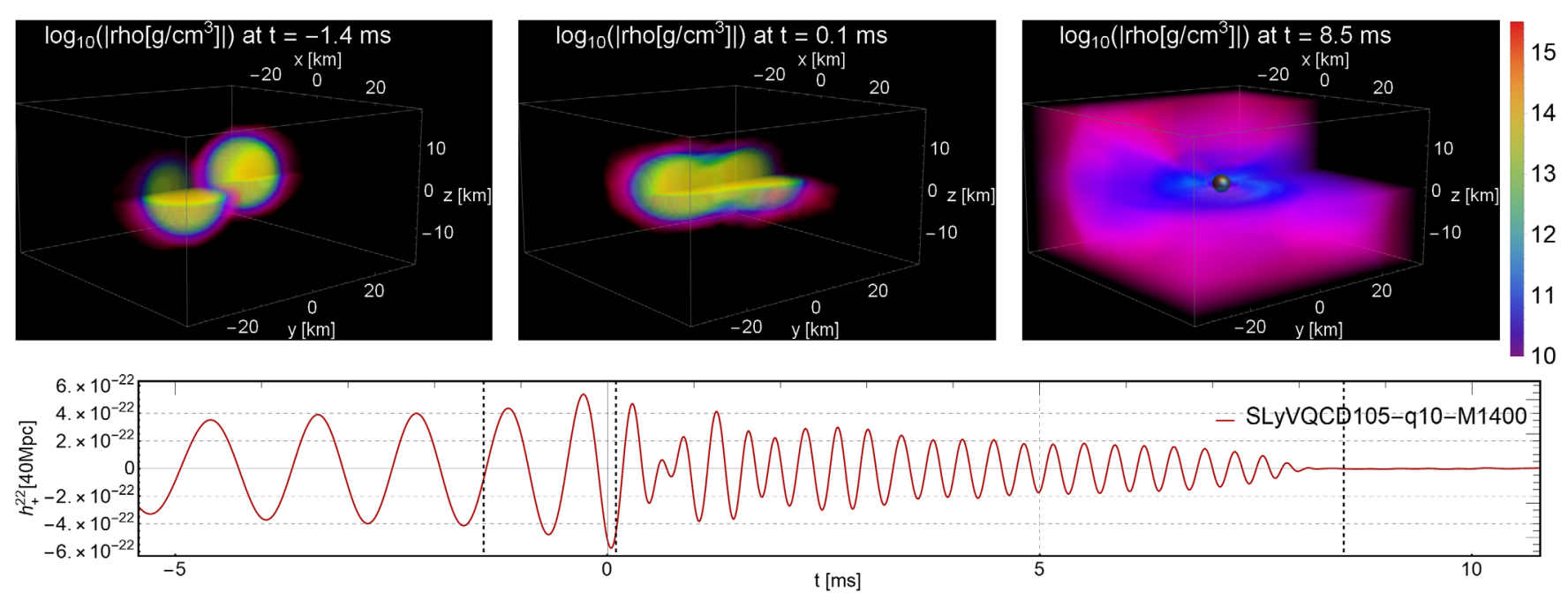

FIG. 4. As in Fig. 3, but shown here for $M=1.4 M_{\odot}$. Snapshots are at the inspiral phase (left), merger phase (center), and after black hole collapse (right). For visibility we omit the densities in one quadrant.

can serve as an indicator for a first-order phase transition. The SLy EoS without a phase transition instead leads to immediate collapse in this case [53].

Figure 5 shows the distribution of densities $d(\rho, t)$ present in the star for several times, defined as $\int d(\rho, t) \mathrm{d} \rho=1$, where $d(\rho, t) \mathrm{d} \rho$ is the fraction of the star that has a density between $\rho$ and $\rho+\mathrm{d} \rho$. Before the collapse the HMNS reaches almost twice the maximum rest-mass density of the original stars. This density is still not enough to reach the quark matter phase (see Fig. 1), so unfortunately the HMNS stars do not explore the phase transition of our hybrid EoSs except right before the collapse to the black hole. Indeed, for the heavier mergers the HMNS can develop densities above the phase transition, but reaching such densities then leads to immediate collapse. In Fig. 6 we show the frame just before an apparent horizon is detected in the simulation of Fig. 4. The heavier cases $\left(M \geq 1.5 M_{\odot}\right)$ lead to immediate collapse after the merger and give no HMNS phase.

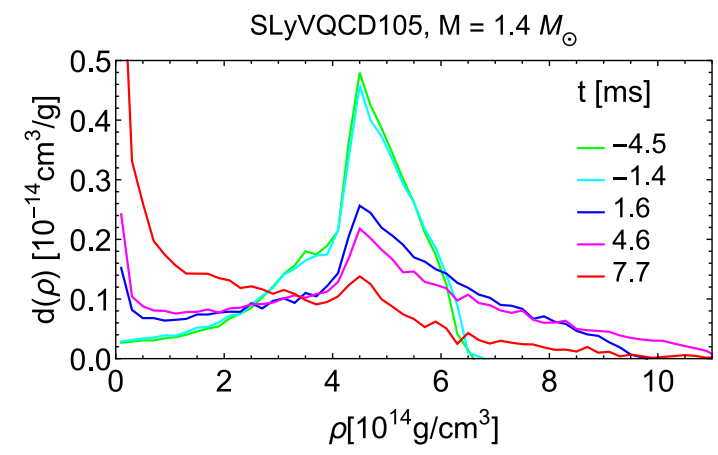

FIG. 5. Distribution of the $\rho$ field of Fig. 4 at different times. After the merger the HMNS reaches a density of about twice the maximum density of the initial star. Shortly after the black hole forms $(t \approx 7.8 \mathrm{~ms})$, most matter is in the black hole with some low-density matter in the surrounding atmosphere.
In Fig. 7 we show the PSDs [Eq. (1)] of our numerical waveforms together with the sensitivity curves of Advanced LIGO (adLIGO) [54] and the Einstein Telescope (ET) $[55,56]$. These spectra show several pronounced features, where the lowest-frequency peak can be attributed to the inspiral phase and the three higher-frequency peaks $\left(f_{1}, f_{2}, f_{3}\right)$ to the post-merger phase. In particular, $f_{2}$ and $f_{3}$ are characteristic for the EoS used [3], whereas the inspiral phase is mostly determined by $M$ and $\Lambda$ [57]. In the left panel we keep $M=1.3 M_{\odot}$ fixed and show our results from two different holographic EoSs with $b=10.5$ (red) and $b=10.6$ (blue) together with the result for standard SLy (green) without input from holography. Increasing $b$ shifts the postmerger peaks toward the higher SLy frequencies, since higher $b$ has a higher matching density (see Table I).

The picture is less clear for the highest-frequency peak $f_{3}$, which in some cases is ambiguous. In contrast to the SLy EoS, we find that the $f_{3}$ peak predicted from our

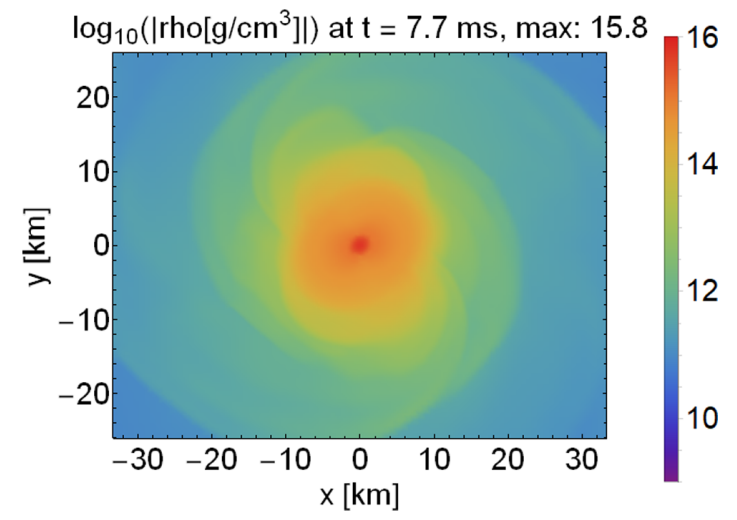

FIG. 6. A snapshot of the simulation presented in Fig. 4 right at the moment before an apparent horizon is detected. The density at the center is larger than $6 \times 10^{15} \mathrm{~g} / \mathrm{cm}^{3}$, which is well into the quark matter regime (see Fig. 1). 

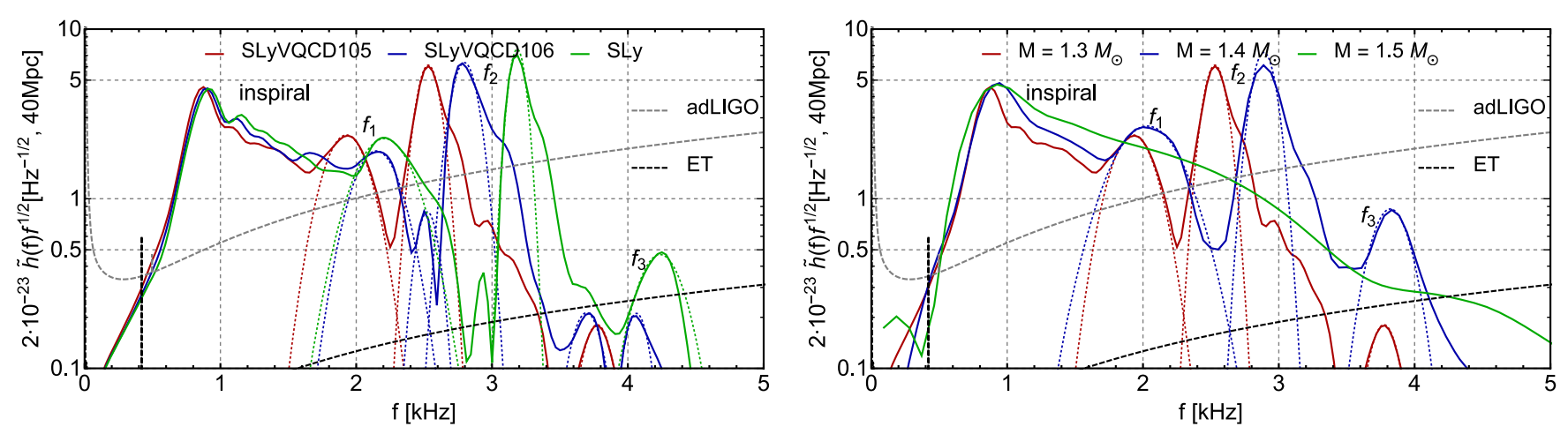

FIG. 7. The PSD for two holographic EoSs with a comparison to the SLy EoS for mergers with $M=1.3 M_{\odot}$ (left) and a comparison of different masses for our holographic EoS with $b=10.5$ (right). The Gaussian fits (dotted curves) determine the characteristic frequencies $f_{1}, f_{2}$, and $f_{3}$ in the post-merger phase, except when $M=1.5 M_{\odot}$, which collapses to a black hole immediately. Black and gray dashed lines are sensitivity curves of the Advanced LIGO (adLIGO) detector [54] and the Einstein Telescope (ET) [55].

holographic EoS will probably not be visible to thirdgeneration detectors for $M=1.3 M_{\odot}$ binaries. In the right panel Fig. 7 we show the PSD for fixed holographic EoS $(b=10.5)$ and three different binary masses. The $M=$ $1.5 M_{\odot}$ case leads to immediate collapse without the HMNS phase and thus to a featureless PSD. For increasing mass, which in the considered mass range results in an increase of compactness, the characteristic frequencies are shifted to higher values. Furthermore, the $M=1.4 M_{\odot}$ case (in contrast to $M=1.3 M_{\odot}$ ) gives a distinguished $f_{3}$ peak that lies clearly above the sensitivity curve of ET.

Finally, in Table II we list the values for $f_{1}, f_{2}$, and $f_{3}$ we extracted from our simulations using a Gaussian fit of the eight frequency points around the local maxima of the peaks.

The values of $f_{1}$ and $f_{2}$ we obtain using the pure SLy EOS are close to those presented in Table III in Ref. [13], where the authors found $f_{1}=2.13$ and $f_{2}=3.23 \mathrm{kHz}$. On the other hand, our values for the holographic EOSs are significantly below the pure SLy values, which leads to the opportunity to distinguish these EOSs using the GW power spectrum, except for the $b=10.6$ case where our $f_{1}=$ $2.15 \mathrm{kHz}$ is close to the pure SLy result $(2.13 \mathrm{kHz})$ of Ref. [13].

The values of $f_{1}$ we find for the pure SLy and our hybrid EoS with $b=10.5$ are in good agreement with the universal curve proposed in Ref. [12]. Indeed, this universal curve predicts a shift to higher frequencies for softer EoSs (increasing $b$ in our case), giving more compact stars (see Fig. 2). Our values for $f_{2}$, together with the corresponding values for $\Lambda$ stated in Table II, can be directly compared to Table II of Ref. [58] which quotes $f_{2}=3.13 \mathrm{kHz}$ and Table I of Ref. [59] which quotes $f_{2}=3.16 \mathrm{kHz}$.

Such a comparison shows that our result for $M=1.4 M_{\odot}$ lies essentially on top of these fitting curves, where our results for $M=1.3 M_{\odot}$ and $M=1.35 M_{\odot}$ reside close to the lower bound of the $2 \sigma$ confidence band. Here we also note that Ref. [58] did an elaborate study on the feasibility of comparing $f_{2}$ obtained from numerical simulations to actual data from advanced gravitational-wave detectors, whereby both detector uncertainties (signal-to-noise ratios) as well as modeling uncertainties were all propagated in order to arrive at an estimate of the achievable accuracy of an $f_{2}$ measurement. They concluded that even with a high measurement accuracy the $f_{2}$ is still roughly $100 \mathrm{~Hz}$ uncertain (in particular, for the SLy4 EOS) since a numerical convergence study is often impossible and modeling of waveforms has its own uncertainties as well.

\section{DISCUSSION}

The characteristic frequencies $\left(f_{1}, f_{2}, f_{3}\right)$ of our holographic EoS from the HMNS phase of the neutron star mergers establish a novel approach to confront predictions from holography with future observational data.

TABLE II. Summary of simulation results. Ambiguous frequencies are written in brackets.

\begin{tabular}{lcccccc}
\hline \hline$M\left[M_{\odot}\right]$ & EoS & $b$ & $f_{1}[\mathrm{kHz}]$ & $f_{2}[\mathrm{kHz}]$ & $f_{3}[\mathrm{kHz}]$ & $\Lambda$ \\
\hline 1.30 & SLyVQCD105 & 10.5 & 1.93 & 2.53 & 3.77 & 820 \\
1.30 & SLyVQCD106 & 10.6 & 2.15 & 2.80 & $3.70(4.06)$ & 620 \\
1.30 & SLy & $\ldots$ & 2.21 & 3.19 & 4.24 & 480 \\
1.35 & SLyVQCD105 & 10.5 & 1.95 & 2.60 & $3.53(3.90)$ & 670 \\
1.40 & SLyVQCD105 & 10.5 & 2.03 & 2.89 & 3.82 & 550 \\
1.50 & SLyVQCD105 & 10.5 & $\cdots$ & $\cdots$ & $\cdots$ & 370 \\
\hline \hline
\end{tabular}


Our results from the holographic model predict a shift of $f_{1}$ and $f_{2}$ to significantly lower frequencies compared to the nonholographic SLy model, which can be attributed to the higher stiffness of our hybrid EoS. Indeed, a measurement of $f_{2}$ significantly larger than $2.8 \mathrm{kHz}$ for a $1.3 M_{\odot}$ merger would clearly exclude our holographic EoS. Furthermore, the holographic EoS predicts that $f_{3}$ is best visible for masses around $1.4 M_{\odot}$, as its amplitude is too low for lower masses, and higher masses lead to quick black hole formation. The highest densities we found in the HMNS phase are about $1.1 \times 10^{15} \mathrm{~g} / \mathrm{cm}^{3}$ (see Fig. 5), except in the center for a period less than $0.5 \mathrm{~ms}$ before the detection of the apparent horizon (see Fig. 6).

The present work can be seen as a first step towards holographic GW model building and is extendable in many different ways. Important next steps are to improve the holographic model by going beyond the homogeneous approach for the V-QCD baryon field and also to systematically study other nuclear matter models than SLy used for the low-density part in this work. Due to limited computing resources we used rather coarse meshes in our simulations, which is something we plan to improve on in future works. More challenging extensions are to include finite-temperature effects in the EoS and magnetic fields in the merger simulations. It would also be exciting to study neutrinos and electromagnetic radiation in this current age of multimessenger astronomy.

\section{ACKNOWLEDGMENTS}

It is a pleasure to thank Umut Gürsoy, Takaaki Ishii, Aleksi Kurkela, Raimond Snellings, and Stefan Vandoren for interesting discussions. C. E. thanks Niko Jokela and Aleksi Vuorinen for motivating this project. We especially thank Elias Most for introducing us to WHISKYTHC and Helvi Witek for invaluable support with the Einstein Toolkit. W. S. gratefully acknowledges the hospitality of the CERN Theory group. This work is partially supported by the Netherlands Organisation for Scientific Research (NWO) under the Grants No. 680-47-518 (Vidi) and No. 680-47-458 (W. S., Veni) and the Delta-Institute for Theoretical Physics ( $\Delta$-ITP), both funded by the Dutch Ministry of Education, Culture and Science (OCW). This work was carried out on the Dutch national e-infrastructure with the support of SURF Cooperative.
[1] B. P. Abbott et al. (LIGO Scientific, Virgo Collaborations), Phys. Rev. Lett. 116, 061102 (2016).

[2] B. Abbott et al. (LIGO Scientific, Virgo Collaborations), Phys. Rev. Lett. 119, 161101 (2017).

[3] L. Baiotti and L. Rezzolla, Rep. Prog. Phys. 80, 096901 (2017).

[4] S. Gandolfi, J. Carlson, and S. Reddy, Phys. Rev. C 85, 032801 (2012).

[5] T. Gorda, A. Kurkela, P. Romatschke, M. Säppi, and A. Vuorinen, Phys. Rev. Lett. 121, 202701 (2018).

[6] E. Annala, T. Gorda, A. Kurkela, J. Nättilä, and A. Vuorinen, arXiv:1903.09121.

[7] E. Annala, T. Gorda, A. Kurkela, and A. Vuorinen, Phys. Rev. Lett. 120, 172703 (2018).

[8] E. R. Most, L. R. Weih, L. Rezzolla, and J. SchaffnerBielich, Phys. Rev. Lett. 120, 261103 (2018).

[9] E. R. Most, L. J. Papenfort, V. Dexheimer, M. Hanauske, S. Schramm, H. Stöcker, and L. Rezzolla, Phys. Rev. Lett. 122, 061101 (2019).

[10] A. Bauswein, N.-U. F. Bastian, D. B. Blaschke, K. Chatziioannou, J. A. Clark, T. Fischer, and M. Oertel, Phys. Rev. Lett. 122, 061102 (2019).

[11] F. Loffler et al., Classical Quantum Gravity 29, 115001 (2012).

[12] K. Takami, L. Rezzolla, and L. Baiotti, Phys. Rev. Lett. 113, 091104 (2014).

[13] K. Takami, L. Rezzolla, and L. Baiotti, Phys. Rev. D 91, 064001 (2015).
[14] D. Radice, S. Bernuzzi, W. Del Pozzo, L. F. Roberts, and C. D. Ott, Astrophys. J. 842, L10 (2017).

[15] F. Maione, R. De Pietri, A. Feo, and F. Löffler, Phys. Rev. D 96, 063011 (2017).

[16] M. Järvinen and E. Kiritsis, J. High Energy Phys. 03 (2012) 002.

[17] U. Gursoy and E. Kiritsis, J. High Energy Phys. 02 (2008) 032.

[18] F. Bigazzi, R. Casero, A. L. Cotrone, E. Kiritsis, and A. Paredes, J. High Energy Phys. 10 (2005) 012.

[19] R. Casero, E. Kiritsis, and A. Paredes, Nucl. Phys. B787, 98 (2007).

[20] T. Alho, M. Järvinen, K. Kajantie, E. Kiritsis, and K. Tuominen, J. High Energy Phys. 01 (2013) 093.

[21] T. Alho, M. Järvinen, K. Kajantie, E. Kiritsis, C. Rosen, and K. Tuominen, J. High Energy Phys. 04 (2014) 124; 02 (2015) 033(E).

[22] D. Areán, I. Iatrakis, M. Järvinen, and E. Kiritsis, J. High Energy Phys. 11 (2013) 068.

[23] M. Järvinen, J. High Energy Phys. 07 (2015) 033.

[24] U. Gursoy, E. Kiritsis, L. Mazzanti, and F. Nitti, Nucl. Phys. B820, 148 (2009).

[25] N. Jokela, M. Järvinen, and J. Remes, J. High Energy Phys. 03 (2019) 041.

[26] T. Ishii, M. Järvinen, and G. Nijs, J. High Energy Phys. 07 (2019) 003.

[27] P. M. Chesler, N. Jokela, A. Loeb, and A. Vuorinen, Phys. Rev. D 100, 066027 (2019). 
[28] C. Hoyos, D. Rodríguez Fernández, N. Jokela, and A. Vuorinen, Phys. Rev. Lett. 117, 032501 (2016).

[29] M. Rozali, H.-H. Shieh, M. Van Raamsdonk, and J. Wu, J. High Energy Phys. 01 (2008) 053.

[30] S.-w. Li, A. Schmitt, and Q. Wang, Phys. Rev. D 92, 026006 (2015).

[31] P. Haensel and B. Pichon, Astron. Astrophys. 283, 313 (1994), https://ui.adsabs.harvard.edu/abs/1994A\%26A.. .283..313H/abstract.

[32] F. Douchin and P. Haensel, Astron. Astrophys. 380, 151 (2001).

[33] J. Antoniadis et al., Science 340, 1233232 (2013).

[34] N. K. Glendenning, Compact Stars: Nuclear Physics, Particle Physics, and General Relativity (Springer, New York, 1997).

[35] J. Nättilä, A. W. Steiner, J. J. E. Kajava, V. F. Suleimanov, and J. Poutanen, Astron. Astrophys. 591, A25 (2016).

[36] J. Nättilä, M. C. Miller, A. W. Steiner, J. J. E. Kajava, V. F. Suleimanov, and J. Poutanen, Astron. Astrophys. 608, A31 (2017).

[37] B. P. Abbott et al. (LIGO Scientific, Virgo Collaborations), Phys. Rev. Lett. 121, 161101 (2018).

[38] H. T. Cromartie et al., Nat. Astron. 4, 72 (2020).

[39] A. Bauswein, H.-T. Janka, and R. Oechslin, Phys. Rev. D 82, 084043 (2010).

[40] E. Gourgoulhon, P. Grandclement, K. Taniguchi, J.-A. Marck, and S. Bonazzola, Phys. Rev. D 63, 064029 (2001).

[41] L. Rezzolla and O. Zanotti, Relativistic Hydrodynamics, edited by L. Rezzolla and O. Zanotti (Oxford University Press, Oxford, 2013). ISBN-10: 0198528906; ISBN-13: 978-0198528906.

[42] E. Schnetter, S. H. Hawley, and I. Hawke, Classical Quantum Gravity 21, 1465 (2004).

[43] J. Thornburg, Classical Quantum Gravity 21, 743 (2004).
[44] T. Goodale, G. Allen, G. Lanfermann, J. Massó, T. Radke, E. Seidel, and J. Shalf, in Vector and Parallel ProcessingVECPAR'2002, 5th International Conference, Lecture Notes in Computer Science (Springer, Berlin, 2003).

[45] F. Banyuls, J. A. Font, J. M. Ibanez, J. M. Marti, and J. A. Miralles, Astrophys. J. 476, 221 (1997).

[46] D. Radice and L. Rezzolla, Astron. Astrophys. 547, A26 (2012).

[47] D. Radice, L. Rezzolla, and F. Galeazzi, Mon. Not. R. Astron. Soc. 437, L46 (2014).

[48] D. Radice, L. Rezzolla, and F. Galeazzi, Classical Quantum Gravity 31, 075012 (2014).

[49] D. Radice, L. Rezzolla, and F. Galeazzi, ASP Conf. Ser. 498, 121 (2015), http://aspbooks.org/a/volumes/article_details/? paper_id=37437.

[50] D. Radice and L. Rezzolla, Astron. Astrophys. 547, A26 (2012).

[51] Movies of the mergers can be found at sites.google.com/site/ wilkevanderschee/neutron-stars.

[52] L. R. Weih, M. Hanauske, and L. Rezzolla, arXiv:1912.09340.

[53] K. Hotokezaka, K. Kyutoku, H. Okawa, M. Shibata, and K. Kiuchi, Phys. Rev. D 83, 124008 (2011).

[54] L. Barsotti, S. Gras, M. Evans, and P. Fritschel, LIGO Document No. T1800044-v5, 2018.

[55] S. Hild et al., Classical Quantum Gravity 28, 094013 (2011).

[56] In our plots we use the ET_D data provided at http://www .et-gw.eu/index.php/etsensitivities.

[57] J. S. Read, L. Baiotti, J. D. E. Creighton, J. L. Friedman, B. Giacomazzo, K. Kyutoku, C. Markakis, L. Rezzolla, M. Shibata, and K. Taniguchi, Phys. Rev. D 88, 044042 (2013).

[58] M. Breschi, S. Bernuzzi, F. Zappa, M. Agathos, A. Perego, D. Radice, and A. Nagar, Phys. Rev. D 100, 104029 (2019).

[59] K. W. Tsang, T. Dietrich, and C. Van Den Broeck, Phys. Rev. D 100, 044047 (2019). 